\title{
reviscafuences
}

ISSN: $1575-7072$ | $6.155 \mathrm{~N}: 2172.7775$

Páginas: 296-305

Recibido: 2020-03-05

Revisado: 2021-04-30

Aceptado: 2021-05-11

Preprint: 2021-09-15

Publicación Final: 2021-09-15

www.revistascientificas.us.es/index.php/fuentes/index

DOI: https://doi.org/10.12795/revistafuentes.2021.11389

\section{Los diarios de aula: una herramienta critico-reflexiva para aprender cuestiones de género}

\author{
Classroom journals: a critical reflective tool for learning about gender issues
}

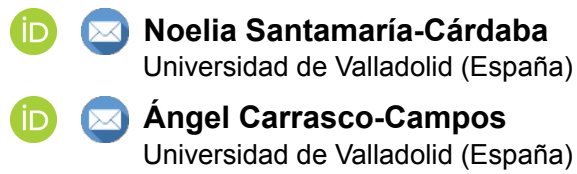

\section{Resumen}

Este artículo analiza una experiencia didáctica en la que se emplean como herramienta didáctico-reflexiva los diarios de aula sobre una cuestión tan relevante en la actualidad como es el quinto Objetivo del Desarrollo Sostenible incluido en la Agenda 2030 promovida por la ONU: el género. Específicamente, este estudio trabaja la desigualdad de género para despertar conciencias crítico-reflexivas en el alumnado. La formación de una ciudadanía global concienciada con las desigualdades y que actúe a favor de la justicia social es imprescindible en la sociedad actual; al igual, que formar, desde los centros educativos, estudiantes que posean una adecuada alfabetización mediática que les haga valorar de forma crítica las noticias provenientes de los medios de comunicación. La investigación empleó un enfoque cualitativo mediante el análisis de los discursos del alumnado y de la maestra generados en los diarios de aula. Los resultados desvelan no solo que la experiencia didáctica resulta exitosa, sino que el empleo del diario en el aula resulta eficaz para formar una ciudadanía global que piense críticamente y comprenda la importancia de actuar contra las desigualdades. En conclusión, emplear como recurso didáctico el diario y las noticias publicadas en medios de comunicación son unas herramientas útiles y eficaces que los docentes pueden aplicar en el aula de forma exitosa, pues de este modo se promueve tanto la formación de ciudadanos globales críticos como la alfabetización mediática.

\section{Abstract}

This article analyses a teaching experience in which learner diaries are used as a reflective teaching tool. This experience focuses on gender: a relevant issue promoted as the fifth objective of the UN 2030 Agenda for Sustainable Development. Specifically, this study works on gender inequality in order to raise critical-reflective awareness among students. The formation of a global citizenship that is aware of inequalities and that acts in favor of social justice is essential in today's society; as is the formation, from educational centers, of students who possess adequate media literacy that makes them critically evaluate the news coming from the media. A qualitative approach has been developed to analyze the discourses that the teacher and the students included on their personal diaries. The results reveal not only that the teaching experience was successful, but also that the use of the diaries is effective in forming a global citizenship that thinks critically and understands the importance of acting against inequalities. In conclusion, the use of the newspaper and news media as a teaching resource is a useful and effective tool that teachers can apply successfully in the classroom, thus promoting both the formation of critical global citizens and media literacy.

\section{Palabras clave}

diario de aula, género, pensamiento crítico, experiencia didáctica, educación primaria, educación, educación rural, educación global.

\section{Keywords}

classroom diary, gender, critical thinking, teaching experience, primary education, education, rural education, global education. 


\section{Introducción}

El empleo de los diarios para promover la reflexión de alumnos y docentes resulta una herramienta con un gran potencial didáctico (Corsi, 2017). Los estudiantes ven fortalecido su proceso de aprendizaje al escribir de forma reflexiva sobre los contenidos que ha ido adquiriendo puesto que tienden a profundizar en las cuestiones que les han resultado más significativas, lo cual puede potenciar la capacidad investigadora (Goldoni, 1996). Asimismo, esta herramienta es eficaz para el desarrollo del Pensamiento Crítico (PC), entendido como la capacidad de "alcanzar la mejor explicación para un hecho, fenómeno o problema con el fin de saber resolverlo eficazmente" (Saiz, 2017, p.19), tal como desarrollaremos más adelante. Las reflexiones que el alumnado va desarrollando en los diarios promueven el PC, pues este "facilita el cuestionamiento de lo que hacemos y/o dejamos de hacer, asimismo, pone en evidencia las diversas formas en que cada uno logra resultados comunes" (Goldoni, 1996, p.68). Igualmente, y de acuerdo con Morduchowicz (1995), los diarios benefician la formación de ciudadanos democráticos, lo cual resulta imprescindible en la sociedad global de la información actual.

En línea con este propósito de contribuir a una ciudadanía democrática, las escuelas deben asumir el reto de formar ciudadanos globales que piensen de forma crítica y sean capaces de actuar frente a las desigualdades. Ante esta situación, el trabajo dentro del horizonte de los Objetivos del Desarrollo Sostenible (ODS) marcados por la ONU en la Agenda 2030 resulta una oportunidad, especialmente si son abordados desde una perspectiva que combine lo local y global que favorezca la erradicación de las desigualdades. La experiencia didáctica que presentamos en este artículo se centra en el trabajo con el quinto de los ODS: la igualdad de género. De acuerdo con Azorín (2017), la escuela debe actuar de forma comprensiva hacia el desafío que supone la globalización social y mediática y colaborar con la erradicación de desigualdades, entre ellas la relativa al género. De hecho, la continuidad de los estereotipos de género en la sociedad actual está "dando lugar a un renovado interés por el estudio de las actitudes hacia la igualdad entre mujeres y hombres" (Azorín, 2017, p.47). Pese a la relevancia de esta temática, Dotti y Quaranta (2017) señalan la escasez de investigaciones sobre las actitudes de niños y jóvenes ante los roles de género, a pesar de que existen múltiples estudios sobre esta cuestión en las personas adultas. Una posible explicación de esta carencia podría situarse en la dificultad de abordar este tipo de cuestiones con estudiantes de corta edad. Por tanto, la igualdad de género es un reto social e institucional para conseguir a nivel global, lo cual hace indispensable que se aborde como algo prioritario en los currículos educativos.

En base a lo establecido en líneas anteriores, el presente estudio tiene como objetivos: 1) Analizar la experiencia didáctica realizada para comprobar las posibilidades del uso de noticias de diarios digitales y del diario como recurso didáctico para la formación de ciudadanos globales críticos; 2) Examinar las percepciones que los estudiantes han reflejado en sus diarios sobre las desigualdades de género tratadas en el aula mediante el empleo de dichas noticias; y 3) desarrollar una valoración crítica de la propuesta analizada, con el propósito de establecer diversas recomendaciones para futuras experiencias. Por ello, este artículo supone una aportación relevante, pues se propone analizar las posibilidades del uso de noticias sobre género y su posterior reflexión en los diarios como recursos para educar ciudadanos críticos y reflexivos que actúen ante las desigualdades.

\section{Fundamentación teórica}

\subsection{El diario como herramienta didáctica}

Los diarios de clase ayudan a que los estudiantes sean autónomos en su proceso de conocimiento al expresar lo que consideran significativo y que profundicen en sus reflexiones al considerar tanto sus pensamientos personales como los que han surgido en el aula por parte de sus compañeros y del docente (Goldoni, 1996). El uso del diario en clase está presente en diversos estudios. En el nivel de la Educación Universitaria se aprecian investigaciones sobre el empleo del diario (Acocella, 2005) en las cuales se llega a la conclusión de que es una herramienta que potencia la reflexión y mejora el proceso de autoaprendizaje. A pesar de su gran aplicabilidad, el número de estudios sobre el uso de esta herramienta en niveles educativos inferiores es menor; de este modo, en Educación Secundaria se aprecian estudios como el de Martínez (2018) y en Educación Primaria el realizado por Coral y Lleixá (2013), los cuales llegan a las mismas conclusiones que los realizados en niveles superior.Sin embargo, no se debe olvidar que el diario no sólo es un recurso positivo para el alumnado, sino también para los docentes. Esta idea del profesor reflexivo está adquiriendo relevancia en los últimos años y por ello, el empleo del diario durante las prácticas formativas mejora el proceso de enseñanzaaprendizaje de los futuros docentes (Bermejo y Mellado, 1995). Incluso, en el contexto universitario, los diarios también son una herramienta interesante para realizar investigaciones (Monsalve y Pérez, 2012). 
El potencial de los diarios educativos va más allá de la mera realización de autorreflexiones, pues "el diario, en combinación con la escuela, puede ciertamente mejorar la formación cívica de los alumnos hacia un ciudadano más democrático, mejor informado, más sensible y más participativo" (Morduchowicz, 1995, p.116). Es decir, el diario en la escuela favorece la formación de ciudadanos globales críticos que estén sensibilizados y actúen de forma cívica, pero ¿qué entendemos por Educación para la Ciudadanía Global?

\subsection{La formación de ciudadanos globales críticos}

La sociedad actual requiere la necesidad de formar una ciudadanía global crítica que comprenda su papel como ciudadano en el mundo y su deber de actuar ante cualquier injusticia. Es decir, se busca impulsar una Educación para la Ciudadanía Global (ECG), la cual busca formar personas globales y autónomas que no solamente estén sensibilizadas con los problemas sociales existentes, sino que actúen tratando de cambiar esta situación (Mesa, 2019). Autores como Celorio (2013) y Dieste, Coma y Blasco-Serrano (2019) destacan la importancia de la ECG para transmitir los contenidos de temáticas de la Agenda 2030, la cual es un plan de acción internacional promovido por la ONU. Concretamente, la ECG tiene una especial importancia ante la desigualdad de género, siendo uno de sus principales objetivos la necesidad de "potenciar la mirada crítica de la realidad desde la perspectiva de género, la interculturalidad, la paz y la ecología" (Celorio, 2013, p.240).

Acorde con Oxfam (2018), la ECG conlleva que los contenidos sean trabajados de forma flexible y sean sustentados en tres principios: conocimiento y comprensión (justicia social, equidad...), habilidades (pensamiento crítico, empatía...), y valores y actitudes (compromiso con la inclusión, el Desarrollo Sostenible...). Es por ello por lo que los centros educativos deben impulsar la ECG, pues conlleva la transmisión de valores para promover actitudes solidarias y a favor de la justicia social, lo cual hace necesaria la formación de ciudadanos globales que piensen críticamente en la actual sociedad de la información y la comunicación.

\subsection{Pensamiento crítico y educación mediática}

El uso de periódicos y noticias en el contexto educativo ha sido siempre un recurso habitual para trabajar en el aula, bien como "auxiliar didáctico" y material "de apoyo a determinadas áreas" ya sean de humanidades, ciencias sociales o ciencias naturales (Aguaded y Pérez-Rodríguez, 1993), como dinamizador de trabajos colaborativos a través de talleres de prensa o periódicos escolares como medio para la alfabetización y la promoción de la lectura. Sin embargo, se debe "recordar que este formato empleado por la prensa, en concreto el del periódico diario, está dirigido al público adulto, presentando un contenido comprensible, cercano (del entorno próximo) y manifiesto en el ambiente (sucesos cotidianos curiosos, novedosos o impactantes)" (Ricoy, 2004, p.174). Es por eso por lo que generalmente ha sido empleado como recurso en la educación para adultos (Ricoy, 2002), Bachillerato (Aguaded y Pérez-Rodríguez, 1993), etc. No obstante, como plantea el proyecto global Newspapers in Education, puede ser empleado para todas las edades y niveles educativos (DeRoche, 1981).

La difusión de los medios digitales y el uso de las redes sociales en el consumo de noticias nos sitúa frente a retos que demandan la intervención desde las instituciones y agentes educativos. Problemas como la contrastación de fuentes, la irrupción de las noticias falsas (fake news) en la era de la «posverdad» o la viralización de contenidos como parte de nuevas formas de consumo mediático, son algunas de las cuestiones que, con cada vez más frecuencia, están asumiendo los procesos educativos (Unesco, 2018), especialmente en Educación Superior (Richardson, 2017). Esta desinformación propia de la «posverdad» puede aparecer asociada al género al difundir noticias falsas con "contenido engañoso, que buscan dañar el feminismo" (Herrero-Diz, Pérez-Escolar y Plaza, 2020, p.208). Sin embargo, y como contrapunto, debe mencionarse la accesibilidad que posibilita el acceso en línea a periódicos que favorecen su uso en niveles y entornos educativos cada vez más diversos, como la Educación Primaria y la escuela rural, caso de estudio de este trabajo.

Resulta, por tanto, necesaria la formación ciudadanos globales alfabetizados mediáticamente. Sin embargo, la educación en medios continúa siendo "una asignatura pendiente" (Kendal y McDougall, 2012, p.21). Además, la actual sociedad de la información hace necesario formar una ciudadanía que piense críticamente y posea la capacidad necesaria para valorar la información desde una perspectiva crítica (Gutiérrez y Tyner, 2012). Desde el ámbito de la educación mediática, Puchner, Markowitz y Hedley (2015) tratan de desarrollar el pensamiento crítico (PC) en la ciudadanía, lo cual confirma que los expertos defienden que "la situación de la educación en medios y la competencia crítica no es únicamente posible sino también necesaria, sobre todo en términos del desarrollo del pensamiento crítico de la audiencia" (Ferodov y Levistkaya, 2015, p.107). 
EI PC es una cuestión trabajada en diferentes áreas de conocimiento, lo cual da lugar a la existencia de múltiples interpretaciones. Este estudio se centra en la definición promovida por Vieira (2018), quien comprende el PC como "forma de pensar centrada en la resolución de problemas y la toma de decisiones racional e inteligente" (p.28). El desarrollo del PC conlleva la adquisición de habilidades para formar una ciudadanía capaz de actuar, como se promueve desde la ECG, y comprender críticamente diversos "contextos y situaciones de la vida cotidiana, desde entender el sentido de una noticia [...]; pasando por la toma de decisiones y resolución de problemas personales [...]; hasta a la participación en la toma de decisiones sobre cuestiones públicas" (Vieira, Tenreiro-Vieira y Martins, 2010, p.101-102). Por esta razón, se deben realizar propuestas o diseñar recursos fundamentados en la pedagogía crítica (Giroux, 1997) que los docentes puedan aplicar en sus aulas, lo cual vislumbra la relevancia de las recomendaciones que emergen del presente estudio que trata de formar una ciudadanía global crítica mediante el empleo de las noticias como recurso didáctico.

\section{Metodología}

Como se anticipaba, el propósito de este estudio es analizar los resultados de una experiencia didáctica en la que se trabajan cuestiones de género a través del empleo de noticias en el aula. Durante esta experiencia, el alumnado utilizó como herramienta de aprendizaje un diario en el que fueron anotando sus reflexiones e impresiones sobre las noticias leídas y debatidas en el aula, mediante el análisis, individual y colectivo, guiado por la docente. Por tanto, se ha dispuesto de un material de análisis reflexivo basado en una experiencia real en el aula, de modo que nos es posible profundizar en los resultados, alcance y limitaciones de esta experiencia. Adicionalmente, se examinarán las fortalezas y debilidades de la propuesta de intervención educativa elaborada e implementada por la maestra, para valorar su eficacia y proponer posibles aspectos a mejorar.

\subsection{Participantes y contextualización}

La experiencia didáctica fue realizada en un centro educativo rural situado en una a $70 \mathrm{~km}$ de la capital: Segovia (España). Concretamente, se realizó con el alumnado de tercer curso, es decir, con 18 estudiantes de los cuales solamente cuatro nacieron en España. El número de estudiantes en la escuela rural española, especialmente en el caso de Castilla y León, tiende a ser reducido, lo cual puede ser visto como una desventaja porque los grupos tienden a ser heterogéneos; sin embargo, el reducido número de estudiantes, desde otra perspectiva, puede ser positivo al favorecer el espíritu democrático y las relaciones profesor-alumno (MoralesRomo, 2017; Price y Stewart, 2015).

\subsection{Propuesta didáctica}

La experiencia didáctica fue realizada por la maestra tutora y conllevaba varias fases: primeramente, la docente proporcionó diversas noticias sobre desigualdad de género y laboral para intentar despertar en ellos el sentido crítico. Estas noticias fueron seleccionadas conforme al juicio experto de la docente y, por tanto, no se consideraron cuestiones como el formato de la pieza o la cabecera en la que la noticia fue publicada, sino las posibilidades de uso pedagógico de los materiales conforme a su experiencia. A continuación, la Tabla 1 proporciona una síntesis de las noticias empleadas.

En segundo lugar, los estudiantes leen, razonan e investigan sobre la información proporcionada. Esta lectura se hace en pequeños grupos y, posteriormente, se reflexiona de manera individual y se debate en gran grupo con el resto de sus compañeros con la finalidad de promover un debate reflexivo y enriquecedor. Estas metodologías activas son idóneas para este tipo de experiencias autorreflexivas, como la realizada por Aramburuzabala (2013) sobre justicia social.

En tercer lugar, la docente solicita a los alumnos que reflexionen en sus diarios de campo sobre las cuestiones relativas a la desigualdad de género. Esta maestra proporciona orientaciones sobre las cuestiones que han ido surgiendo en el aula para que puedan reflexionar sobre ellas de forma personal en sus diarios, pues de esta forma se facilita que los estudiantes concentren su atención y reflexionen críticamente sobre los contenidos más significativos.

Por último, la docente refleja en su diario reflexiones sobre la sesión realizada indicando si la sesión funcionó correctamente, qué aspectos han llamado su atención o qué se puede mejorar para futuras ocasiones. 
Tabla 1

Información sobre las noticias empleadas. Fuente: elaboración propia.

\begin{tabular}{|l|l|l|}
$\begin{array}{l}\text { Fecha de publicación } \\
08 / 03 / 2017\end{array}$ & $\begin{array}{l}\text { Titular } \\
\text { Luchar contra la precarización } \\
\text { y explotación laboral hacia las } \\
\text { mujeres }{ }^{4} \\
\text { Mujeres y niños sufren explotación } \\
\text { laboral en } \mathrm{NL}^{5}\end{array}$ & $\begin{array}{l}\text { Breve descripción } \\
\text { La precarización laboral y la } \\
\text { explotación de las personas es algo } \\
\text { que ocurre a diario especialmente } \\
\text { entre mujeres. } \\
\text { La explotación laboral es algo que } \\
\text { afecta tanto a mujeres como, en } \\
\text { ocasiones, a menores. } \\
\text { Desigualdad, crisis económica ha afectado a las } \\
\text { abusos... Explotación laboral } \\
\text { de la mujer trabajadora hoy }\end{array}$ \\
$\begin{array}{l}\text { mujeres viéndose obligadas a padecer } \\
\text { una doble explotación: 1) el cuidado } \\
\text { familiar y del hogar; 2), trabajar en } \\
\text { empresas que no pagan de forma } \\
\text { equitativa. }\end{array}$
\end{tabular}

\subsection{Metodología de investigación}

Este artículo emplea como fuente de información principal los diarios de los estudiantes y el diario de la docente, siendo por tanto los diarios de clase el objeto de este estudio al permitir recopilar datos sobre lo que va aconteciendo en las aulas (Zabalza, 2004). Se propone un análisis cualitativo de los discursos del alumnado y de la maestra generados en sus respectivos diarios, al considerar que "no hay prácticas educativas al margen de una construcción de significaciones" (Buenfil, 1993, p.7-8).

Estos diarios permiten conocer las valoraciones e impresiones de la educadora y los educandos puesto que son un recurso que se emplea para registrar las reflexiones personales de las sesiones desarrolladas en el aula (Vain, 2003). Asimismo, no solo posibilitan recopilar información significativa, sino que permite realizar comparaciones, autoevaluaciones y heteroevaluaciones, ser más conscientes y cambiar actitudes (Jurado, 2011). Para garantizar el anonimato a los participantes, se ha realizado una codificación para analizar cualitativamente los diarios del estudiantado (Taylor y Bogdan, 2010). En este caso, el primer estudiante se corresponde con E1, el segundo con E2 y así sucesivamente; asimismo, se ha incorporado $\mathrm{H}$ si es un alumno y $\mathrm{M}$ si es una alumna.

El procedimiento de análisis empleado por el equipo investigador para sistematizar y analizar la información facilitada en los diarios se articula conforme a las tres categorías con las que se solicitaba a los estudiantes anotar sus reflexiones en sus diarios. Así, por tanto:

1. Desigualdad de género en el ámbito laboral. Los estudiantes reflejan en sus diarios de campo reflexiones relacionadas con la situación de desigualdad laboral que padecen las mujeres.

2. Desigualdad de género Norte-Sur. Se pretendía conocer si el alumnado asociaba, o no, estas situaciones de desigualdad e injusticia a países y entornos de pobreza, y por qué.

3. Actuaciones contra la desigualdad. Como parte de la propuesta de intervención educativa, se proponía al alumnado participar en reflexiones, individuales y grupales, sobre posibles acciones y medidas para evitar este tipo de injusticias.

Finamente, en un ejercicio de transparencia y ética se compartió el análisis de los diarios con la maestra dotando de rigurosidad a este estudio según los críticos de rigor científicos propuestos por Guba (1989). (1989).

\section{Resultados y discusión}

A continuación, se analizan las reflexiones recopiladas en los diarios desarrollados para esta propuesta y en última instancia, se presentan los resultados de la experiencia didáctica.

\subsection{Resultados de los diarios}

\subsubsection{Desigualdad de género en el ámbito laboral}

Respecto a esta primera cuestión analizada, entre las observaciones de los estudiantes encontramos de manera habitual juicios que valoran negativamente las situaciones de desigualdad de las que hablan las 
noticias: "No es justo porque está mal" $(\mathrm{E} 1 \mathrm{H})$, "No es justo porque deben trabajar las mujeres y cobren lo mismo que los hombres" $(\mathrm{E} 14 \mathrm{H})$ o, entre otras, "No porque todos somos iguales" $(\mathrm{E} 11 \mathrm{H})$. En este sentido, la maestra refleja en su diario que el alumnado "no ha visto en mayor medida esa desigualdad. En casa sus padres los dos trabajan y creo yo que no se comenta en casa esta desigualdad". No obstante, al profundizar en esta cuestión con las respuestas que reflejan cuestiones relativas a la carga de trabajo, encontramos diversas reflexiones en los diarios: "No porque las mujeres a veces hacen más que los hombres y ayudan y cobran menos" (E18H), "No porque son ellas las que tienen que hacer todo lo de casa" (E16M) o, entre otras, "No porque las mujeres trabajan más" (E9H). Cabe destacar que mientras que los alumnos se limitan en la mayoría de los casos a reconocer la injusticia, las alumnas dan respuestas en las que se aprecia cierta conciencia o sensibilidad de que las consecuencias de dicha situación así como de la necesidad de actuar por una igualdad en todos los ámbitos para conseguir una sociedad igualitaria; algunas de estas respuestas son: "No porque no es justo que las mujeres sufran y los hombres no" (E2M), "No porque los hombres se creen mejores" (E6M), o "No porque los hombres maltratan a las mujeres" (E12M). Se aprecian, por tanto, valoraciones negativas comunes entre chicos y chicas, pero con un trasfondo diferente. Mientras que los alumnos plantean sus análisis desde el reconocimiento de la desigualdad, especialmente en relación con la descompensación de la carga de trabajo, las alumnas ahondan en las consecuencias sociales y emocionales de dichas situaciones.

Las respuestas de los estudiantes van en la línea de la idea que desprenden Dieckman, Eagly Kulesa (2002), pues afirman que los hombres continúan teniendo más poder debido a que perdura la sociedad patriarcal. Dotti y Quaranta (2017) resaltan que esta desigualdad sigue existiendo, por ejemplo: en el trabajo, el salario o el reparto de tareas domésticas. Incluso, Arvayo y Rodríguez (2015) investigan sobre los roles de género presentes en el alumnado de los tres últimos cursos de Educación Primaria y, al igual que en esta investigación, comprueban que la igualdad entre género es deficiente. Por su parte, Puchner, Markowitz y Hedler (2015) realizaron un estudio que buscaba conocer si los estudiantes expuestos a una educación mediática crítica sobre estereotipos de género a través de los medios de comunicación tenían mayor predisposición a pensar que las mujeres sufren discriminación laborar que si no hubieran recibido esta formación. Los resultados, al igual que los del presente estudio, muestran que el alumnado que recibe una correcta formación mediática crítica se muestra más sensibilizado ante estas situaciones de injusticia.

\subsubsection{Desigualdad de género Norte-Sur}

En este caso, todos los estudiantes señalan en sus respuestas que no solo se dan estas situaciones de desigualdad de género en los países empobrecidos, sino que también se dan en los países más ricos. Las respuestas muestran, al igual que en la categoría anterior, mayor profundidad en el caso de las proporcionadas por las alumnas. Algunas evidencias de las observaciones anotadas por los varones son: "No porque en los países pobres tienen que trabajar también los hombres no solo las mujeres también los hombres" (E2H), o "No solo es en los países pobres se reparten estas injusticias, también en los países ricos" (E14H). Por su parte, las alumnas reflejan en sus diarios respuestas como: "No solo en los países pobres, también en los ricos se violan, no se las deja trabajar, las pagan menos y somos iguales todos" (E5M), "No porque maltratan a las mujeres, las violan y las pegan los hombres" (E6M) o "No porque maltratan hasta morirse" (E16M). Asimismo, ante esta cuestión, la maestra señala en su diario de campo que "la mayoría de los niños ponen el énfasis en el maltrato de la mujer. Critican esta situación y son consciente de ello, les impacta observar noticias de este tipo". De nuevo, se percibe un componente más emocional y reivindicativo en las respuestas de las alumnas al tratar cuestiones como el sufrimiento, el maltrato y las violaciones, mientras que los alumnos se centran en cuestiones materiales, por ejemplo, al exponer que "los hombres trabajan en las oficinas y las mujeres están en casa y ellas no tienen mucho dinero y los hombres sí" (E18H). Así, aun cuando los estudiantes reflejan sensibilidad al justificar que las desigualdades de género pueden ocurrir indistintamente en entornos socioeconómicos diversos, los resultados del trabajo con periódicos revelan que ayudan a fomentar una ciudadanía global en el marco del PC, condiciones indispensables para formar ciudadanos sensibilizados y conscientes de la realidad social. Por ello, como se ha comprobado, resulta positivo tratar con los estudiantes que en determinados países "la marginación y la explotación por género, clase y raza produce un efecto sumatorio en la discriminación y una peculiar combinatoria de simbolismos de dominación" (Puelo, 2000, p.88).

Por otro lado, las reflexiones ligadas a la violencia de género que sufre la mujer por parte del hombre transmiten un mensaje a la sociedad, pues revela que el hombre tiende a actuar de esta manera para tratar de "demostrar y apuntalar la posición de superioridad" (Lorente, 2007, p.25). Unesco (2019) señala que las noticias sobre violencia de género suelen presentar a las mujeres no sólo como víctimas, sino como responsables de sufrir estas agresiones, pues los agresores no suelen formar parte de los informes de las noticias siendo, tratadas como violencia a nivel doméstico y no como un problema que afecta a la estructura social. En esta línea se aprecian investigaciones, como la realizada en las escuelas públicas de Galicia (España), que reflejan la preocupación de los directores escolares, pues consideran la educación en igualdad totalmente necesaria 
para evitar injusticias y la violencia de género dado que "tales valores no han sido totalmente interiorizados por niños, niñas y jóvenes, y por ello desde la escuela debemos continuar educando con una perspectiva de género" (Valle, 2015, p.339).

Cabe destacar que el profesorado debe poseer una formación adecuada para transmitir valores de igualdad y respeto en la escuela para ayudar a que el alumnado desarrolle su PC y actúe ante las desigualdades de cualquier tipo (Peach y Clare, 2017). Esta falta de formación del profesorado en educación mediática crítica quizá sea el detonante de que en el ámbito de la educación formal haya «escasez de contenidos y prácticas de aprendizaje, que podrían ampliarse y mejorarse» (Cabero y Guerra, 2011, p.110) para formar ciudadanos responsables y activos tanto con la sociedad, como con el consumo mediático.

\subsubsection{Actuaciones contra las desigualdades}

La desigualdad laboral de género también fue trabajada en el aula, reflexionando de forma individual y grupal sobre qué se podría hacer para evitar estas injusticias. Las anotaciones de los diarios muestran aquí también diferencias entre los alumnos y las alumnas, pues los varones se limitan a afirmar que la solución es "que paguen lo mismo a las mujeres que a los hombres" (E3H) o, con otras palabras, "dar el mismo dinero" (E9H). Las alumnas añaden que para evitar estas desigualdades se deben realizar "muchas cosas, pero en caso que pagaran lo mismo a las mujeres" (E4M) o, como indica E5M, es necesario "que el hombre también trabaje en casa, que se paguen lo mismo y puedan trabajar en lo mismo, no haya peleas o violencia porque todos somos iguales" (E5M). Estas respuestas relacionadas con el reparto de las tareas domésticas también se encuentran en otras que señalan que la solución a la desigualdad es "que los hombres también colaboren en casa" (E16M), o "que los hombres colaboren más en casa" (E7H, como excepción entre las reflexiones de los varones).

Los resultados sobre las acciones contra las desigualdades muestran, nuevamente, una diferenciación entre géneros, la cual se ve reflejada en la repartición de las tareas del hogar, pues generalmente las reflexiones de los estudiantes resaltaban que los hombres debían participar en dichas labores. Kalacy y Hayrserver (2014) afirman que la mayoría de los estudios indican que las características de los hombres no sufren cambios debido a que están socialmente aceptadas, e incluso se conciben como deseadas. En este sentido, la igualdad educativa debería enfocarse de forma que "no pasara por la adaptación a intereses, actitudes, posiciones y representaciones sociales marcadas por el modelo masculino" (Flecha, 2014, p.58). Finalmente, se debe señalar que la escuela como institución tiene un papel fundamental para mostrar sensibilidad ante estas temáticas, pues si la motivación proviene de todo el centro educativo adquirirá mejores resultados que si los docentes trabajan de forma aislada (Blanco, 2006).

\subsection{Resultados de la experiencia didáctica}

Por último, resulta imprescindible realizar una valoración crítica de esta experiencia, pues a pesar de que funcionó satisfactoriamente y los estudiantes reflexionaron y profundizaron en sus diarios sobre las cuestiones de género tratadas en el aula, deben destacarse diversos aspectos a mejorar. Por un lado, los puntos fuertes de esta experiencia son claros, pues no solo promueve el desarrollo del PC, sino que consigue formar ciudadanos globales críticos que defiendan una sociedad justa, equitativa y humana. Por otro lado, las limitaciones que se encontraron fueron la falta de formación del docente en aspectos como la selección de noticias, el reducido número de alumnos y la necesidad de adaptarse a los horarios marcados por el centro sin poder extenderse en otras asignaturas de manera transversal. Entre estas limitaciones, la heterogeneidad del aula y el reducido número de alumnos son propias de la escuela rural (Price y Stewart, 2015).

En base a esta valoración crítica, las recomendaciones de mejora para futuras experiencias didácticas son:

1. La formación del profesorado en ECG y en educación mediática para impulsar el PC en el aula, puesto que deben aprender para ser agentes de cambio y trabajar en el aula cuestiones globales de forma adecuada para desarrollar la conciencia crítica de sus estudiantes (Martínez-Lirola, 2020).

2. La sistematización en la selección de las noticias puesto que, aunque el juicio experto del docente debe ser un criterio indispensable, se sugieren alternativas como: i) el desarrollo del PC al escoger las fuentes, y la participación del alumnado y las familias en dicha selección; ii) o que el docente procure mayor diversidad en el tipo de pieza escogida (sean noticias, reportajes...) así como la cabecera (para incluir diversidad de líneas editoriales).

3. El compromiso de completar el diario del docente, pues resulta una herramienta beneficiosa que bien realizado permite realizar análisis evaluativos del desarrollo de la experiencia didáctica (Fuentes, 2003).

4. Utilizar las noticias como recurso didáctico para tratar en el aula temáticas sociales mediante enfoques comprensivos buscando fomentar una reflexión individual y grupal. 
5. La sensibilización de los centros educativos ante estas cuestiones, para impulsar el trabajo en equipo promoviendo el trabajo cooperativo y la realización de debates entre estudiantes de diversos niveles para solventar el limitado número de estudiantes por aula en la escuela rural.

\section{Conclusiones}

A lo largo de este estudio, se ha comprobado que los resultados de la experiencia didáctica realizada, en la cual se buscaba trabajar cuestiones de género mediante el empleo de noticias en el aula y del uso del diario como herramienta crítico-reflexiva, han sido positivos. De este modo, el empleo de noticias en el aula ha resultado útil, puesto que el hecho de trabajar con piezas informativas y sucesos reales ha fomentado que los estudiantes se hayan mostrado reflexivos, sensibilizados y críticos tanto ante las situaciones de desigualdad de género como ante otras injusticias sociales. De manera adicional, el análisis de los discursos recopilados en los diarios de campo ha permitido desvelar diferencias en las reflexiones en función del género del alumnado, como se ha ido mostrando en los resultados.

Por otro lado, tanto el reducido número de los estudiantes rurales participantes como su corta edad pueden suponer una limitación. Sin embargo, la necesidad de realizar investigaciones en escuelas rurales y la importancia de la temática tratada hace que este estudio tenga valor para futuras investigaciones relacionadas con el análisis las experiencias, valoraciones y formación de profesores en el uso de noticias como recurso didáctico, conocer otras experiencias en la incorporación de cuestiones globales en su práctica pedagógica cotidianas, e incluso, realizar con alumnos de edades superiores un análisis de la presentación del género en los de medios de comunicación siguiendo los indicadores marcados por la Unesco (2014), entre otras.

Así, el valor del análisis desarrollado si bien puede encontrarse en los resultados alcanzados, podemos situarlo también en la apertura de nuevas líneas de investigación y, de igual modo, en la posibilidad de tomar esta experiencia como un punto de partida, a partir del cual mejorar y ofrecer alternativas para la puesta en práctica en el aula partiendo de los resultados y recomendaciones ya indicados. Por tanto, se puede concluir que emplear como recurso didáctico el diario y las noticias publicadas en medios de comunicación resultan herramientas útiles y eficaces que los docentes pueden aplicar en el aula.

\section{Referencias}

Acocella, C. (2005). Using diaries to assess nonprescription drug use among university students. Journal of Drug Education, 35(4), 267-274. https://doi.org/10.2190/64XK-FDYP-VNQ5-D26M

Aguaded, J., y Pérez-Rodríguez, M. (1993). Prensa y medios de comunicación en Bachillerato. Comunicar, 1, 45-61.

Aramburuzabala, P. (2013). Aprendizaje-servicio: Una herramienta para educar desde y para la justicia social. RIEJS, 2(2), 5-11. https://doi.org/10.15366/riejs2013.2.2

Araya, S. (2004). Hacia una educación no sexista. Revista Electrónica Actualidades Investigativas en Educación, 4(2), 1-13. https://doi.org/10.15517/aie.v4i2.9088

Arvayo, K., y Rodríguez, A. (2015). Roles de género, interacción verbal y tolerancia que presentan alumnos (as) en educación primaria. Revista electrónica de Humanidades, 10(19), 17-31.

Azorín, C. (2017). Actitudes hacia la igualdad de género en una muestra de estudiantes de Murcia. Revista Complutense de Educación, 28(1), 45-60. http://dx.doi.org/10.5209/rev_RCED.2017.v28.n1.48715

Bermejo, M., y Mellado, V. (1995). Los diarios de prácticas en la formación de maestros. REIFOP, 23, 121-136.

Blanco, M. (2006). La equidad y la inclusión social: uno de los desafíos de la educación y la escuela hoy. Revista Iberoamericana sobre Calidad, Eficacia y Cambio en Educación, 4(3), 1-15. Retrieved from https://www.redalyc.org/pdf/551/55140302.pdf

Buenfil, R. (1993). Análisis del discurso y educación. México.

Cabero, J., y Guerra, S. (2011). La alfabetización y formación en medios de comunicación en la formación inicial del profesorado. Educación XX1, 14(1), 89-115. Retrieved from https://www.redalyc.org/pdf/706/70618224004 .pdf

Celorio, G. (2013). Sensibilización y Educación para el Desarrollo. Universidad Jaume I.

Coral, J., y Lleixá, T. (2013). Las tareas en el aprendizaje integrado de educación física y lengua extranjera (AICLE). Retos, 24, 79-84.

Corsi, L. (2017). Diarios escritos y virtuales de diálogo-reflexión. Voces y silencios, 8(1), 144-172. https:// doi.org/10.18175/vys8.1.2017.09

Deroche, E. (1981). Newspapers in education: What we know. Newspaper Research Journal, 2(3), 59-63. https://doi.org/10.1177/073953298100200307

Dieste, B., Coma, T., y Blasco-Serrano, A. (2019). Inclusión de los objetivos de desarrollo sostenible en 
el currículum de Educación primaria y secundaria en escuelas rurales de Zaragoza. RIEJS, 8(1), 97-115. https://doi.org/10.15366/riejs2019.8.1.006

Dotti, G., y Quaranta, M. (2017). The Best Is Yet to Come? Attitudes Toward Gender Roles Among Adolescents in 36 Countries. Sex Roles, 1-2, 30-42. https://doi.org/10.1007/s11199-016-0698-7

Fedorov, A., y Levitskaya, A. (2015). Situación de la educación en medios y la competencia crítica en el mundo actual: opinión de expertos internacionales. Comunicar, 22(45), 107-116.

Flecha, C. (2014). Desequilibrios de género en educación en la España Contemporánea: causas, indicadores y consecuencias. AREAS, 33, 49-60.

Fuentes, E. (2003). El diario escolar: un recurso para la investigación del profesorado. Revista Padres y Maestros, 275, 32-39.

Giroux, H. (1997). Los profesores como intelectuales: hacia una pedagogía crítica del aprendizaje. Barcelona: Paidós.

Goldoni, C. (1996). Un diario para la vida. Revista Abra, 27, 65-72.

Guba, E. G. (1989). Criterios de credibilidad en la investigación naturalista. En Gimeno. In J. Gimeno, A. PérezGómez, y A. (Eds.), La enseñanza: su teoría y su práctica (p. 148-165). Akal.

Gutiérrez, A., y Tyner, K. (2012). Educación para los medios, alfabetización mediática y competencia digital. Comunicar, 19(38), 31-39.

Herrero-Diz, P., Pérez-Escolar, M., y Plaza, J. (2020). Desinformación de género: análisis de los bulos de Maldito Feminismo. Icono14, 18(2), 188-216. http://doi.org/10.7195/ri14.v18i2.1509

Hunt, F. (2012). Global learning in primary schools in england: Practices and impacts.

Indicadores de Género para Medios de Comunicación. (2014). Paris.

Journalism, fake news \& disinformation: handbook for journalism education and training. (2018). Paris.

Jurado, D. (2011). El diario como un instrumento de autoformación e investigación. Revista Qurriculum, 24, 173-200.

Kalayci, N., y Hayrsever, F. (2014). An Analysis of Citizenship and Democracy Education Text Book in the Context of Gender Equality and Determining Students' Perceptions on Gender Equality. Educational Sciences, 14(3), 1065-1074.

Kendall, A., y Mcdougall, J. (2012). Alfabetización mediática crítica en la postmodernidad. Comunicar, 19(38), 21-29.

Lorente, M. (2007). Violencia de género, educación y socialización: acciones y reacciones. Revista de Educación, 342, 19-35.

Martínez, S. (2018). Propuesta de instrumentos de evaluación de la lectura en Secundaria (I): el diario de lectura. Revista e-CO, 15, 143-170.

Martínez-Lirola, M. (2020). Hacia una educación inclusiva: formación del profesorado de primaria enmarcada en los ODS que potencien la igualdad de género. Revista Iberoamericana de Educación, 82(2), 27-45. https://doi.org/10.35362/rie8223596

Mesa, M. (2019). La Educación para la Ciudadanía Global: Una apuesta por la Democracia. RIEJS, 8(1), 15-26. https://doi.org/10.15366/riejs2019.8.1.001

Monsalve, A., y Pérez, E. (2012). El diario pedagógico como herramienta para la investigación. Itinerario Educativo, 26(60), 117-128. https://doi.org/10.21500/01212753.1406

Morduchowicz, R. (1995). El diario y la formación de un ciudadano democrático. Comunicar, 4, 114-117.

Oxfam. (2018). Teaching Controversial Issues: A guide for teachers. Oxford: Author.

Peach, S., y Clare, R. (2017). Global citizenship and critical thinking in higher education curricula and police education. Journal of Pedagogic Development, 7(2), 46-57. Retrieved from http://hdl.handle.net/10547/622145

Price, A., y Stewart, T. (2015). Exploring place and practicing justice: Preparing pre-service teachers for success in rural schools. Journal of Research in Rural Education, 9(30), 1-12.

Puchner, L., Markowitz, L., y Hedley, M. (2015). Critical media literacy and gender: Teaching middle school students about gender stereotypes and occupations. Journal of Media Literacy Education, 7(2), 23-34.

Puelo, A. (2000). Multiculturalismo, educación intercultural y género. Tabanque, 15, 79-91.

Richardson, N. (2017). Fake news and journalism education. Asia Pacific Media Educator, 27(1), 1-9. https:// doi.org/10.1177/1326365X17702268

Ricoy, M. (2002). La educación de adultos y el uso didáctico de la prensa. Comunicar, 19, 184-191.

Ricoy, M. (2004). La prensa en la educación de adultos. Comunicar, 23, 173-180.

Saiz, C. (2017). Pensamiento crítico y cambio. Pirámide.

Setting the Gender Agenda for Communication Policy: New proposals from the Global Alliance on Media and Gender. (2019). Paris.

Taylor, S., y Bogdan, R. (2010). Introducción a los métodos cualitativos de investigación. Barcelona: Paidós. UNESCO. (2014). Indicadores de Género para Medios de Comunicación. París.

UNESCO. (2018). Journalism, fake news \& disinformation: handbook for journalism education and training. París. 
UNESCO. (2019). Setting the Gender Agenda for Communication Policy: New proposals from the Global Alliance on Media and Gender. París.

Vain, P. (2003). El diario académico: una estrategia para la formación de docentes reflexivos. Perfiles educativos, 25(100), 56-68.

Valle, J. (2015). Educando para prevenir la violencia de género desde las aulas: el caso de los centros de educación infantil y primaria (CEIPS) gallegos. Innovación educativa, 25, 327-342.

Vieira, R. (2018). As comunidades online na promoção do pensamento crítico em didática das ciências. Aveiro: UA Editora.

Vieira, R., Tenreiro-Vieira, C., y Martins, I. (2010). Pensamiento crítico y literacia científica. Alambique, 65, 96-103.

Vieira, R., y Vieira, C. (2016). Práticas didático-pedagógicas de ciências: Estratégias de ensino / aprendizagem promotoras do pensamento crítico. Saber \& Educar, 20, 34-41. http://dx.doi.org/10.17346/se.vol20.191

Zabalza, M. (2004). Diarios de clase. Un instrumento de investigación y desarrollo profesional. Madrid: Narcea. 